\title{
Assessing Morphological and Genetic Variation in Annatto (Bixa orellana L.) by Sequence-related Amplified Polymorphism and Cluster Analysis
}

\author{
Ruby Valdez-Ojeda, José Luis Hernández-Stefanoni, \\ Margarita Aguilar-Espinosa, and Renata Rivera-Madrid ${ }^{1}$ \\ Centro de Investigación Cientifica de Yucatán, Unidad de Bioquímica y \\ Biologia Molecular de Plantas, 43 \# 130 Chuburná de Hidalgo CP 97200, \\ Mérida, Yucatán, México
}

\section{Rodomiro Ortiz \\ Centro Internacional de Mejoramiento de Maiz y Trigo, AP 6-641, México, DF, México}

\author{
Carlos F. Quiros \\ Department of Vegetable Crops, University of California, Davis, CA 95616
}

Additional index words. molecular marker, germplasm, bixin, genetic improvement, two-step cluster, AMOVA

\begin{abstract}
Annatto (Bixa orellana L.) is the sole source of bixin, a seed-specific red apocarotenoid pigment used worldwide. Genetic improvement of annatto has focused on increasing bixin content as well as fostering traits that favor higher content such as pod indehiscence and flower color, among others. Eighty-seven samples collected from two separate important agricultural regions of the southeast of Mexico were characterized morphologically and analyzed genetically for the first time. The sequence-related amplified polymorphism was used for genetic analysis. Two-step cluster analysis of the individuals based on morphological traits produced three groups: one containing individuals with desired morphological characteristics and two others with complementary traits. Like the morphological analysis, the genetic analysis indicated high genetic variation, although the dendrogram based on Nei and Li's similarity coefficient showed them to be dispersed by collection site. However, calculated similarity index values indicated all individuals exhibited high genetic variation. The results are an important advance toward a more effective genetic improvement of annatto because crosses between the different morphological and/or genetic groups described here have the potential to produce a recombination of desired traits in hybrid offspring.
\end{abstract}

Annatto (Bixa orellana L.) is a commercial crop originally from the southwest Amazon (Sandy-Cuen and Becerra, 2003). It is grown for its unique capability to produce

\footnotetext{
Received for publication 4 Aug. 2008. Accepted for publication 21 Aug. 2008.

This research was financed by the International Foundation for Science (IFS) F/2932-2 and the Consejo Nacional de Ciencia y Tecnología (CONACYT) 31602B and 46541. Ruby ValdezOjeda received a CONACYT $\mathrm{PhD}$ grant (No. 185874). Dr. Renata Rivera-Madrid and Dr. Carlos F. Quiros received support from UC MexusCONACYT.

We thank Dr. Dan Qiu for technical assistance with the SRAP markers, Dr. Emilio Laca for advice on the cluster analysis, and Dr. Dan Potter for advice on dendrogram construction. Dr. Patricia Colunga and Dr. Jaime Martínez provided academic advice. Mr. Filogonio May participated in the fieldwork. We thank Mr. Julián Coello for technical advices in preparation of acrylamide gels.

${ }^{1}$ To whom reprint requests should be addressed; e-mail renata@cicy.mx
}

Mexico, especially on the Caribbean coast) (Portela de Carvalho et al., 2005). In Mexico, annatto is primarily grown in the southeast states (Sandy-Cuen and Becerra, 2003), particularly in Yucatan, which has the highest annatto production in the country. It is used extensively in regional cuisine and is consequently grown in many agricultural fields and even in backyards.

Annatto has a number of cultigens, including the varieties leiocarpa (Kuntze) and urucurana (Willd) (Poppendieck, 2001), and these manifest wide phenotypic variation in the shape and color of capsules and flowers (Arce, 1999). This species' ecology, cultivation, and industrial applications have been researched previously (RiveraMadrid et al., 2006), but limited effort has been made to classify its cultivars based on bixin content and morphological traits; therefore, such research will facilitate rapid and accurate selection of parental genotypes for further genetic improvement.

Morphological characterization was initially carried out in Venezuela, where variation in traits clustered individuals by origin (Mazzani et al., 2000). Isozyme variation has been studied for annatto in Brazil and indicated that genetic differences corresponded to geographic differences (Portela de Carvalho et al., 2005). Similar research in Venezuela allowed identification of unique genotypes within highly diverse populations (Medina et al., 2001a).

The objective of this study was to analyze morphological variation in $B$. orellana and support it for the first time with genetic variation data generated using molecular markers (SRAP) (Li and Quiros, 2001). High bixin content material and agronomically important traits favoring bixin yield were also evaluated such as pod indehiscence and flower color, among others. The agronomical traits were compared with bixin production and the individuals were analyzed genetically to select the most appropriate germplasm for genetic improvement of annatto.

\section{Materials and Methods}

nantly in the seed aril and widely used in the food industry (Giuliano et al., 2003).

The demand for natural colorants of annatto has increased for its natural nutrients and its beneficial effects in lowering glucose (Russell et al., 2005) and cholesterol (McGonigle and McCracken, 2002) in blood. It has also displayed antimutagenic (Júnior et al., 2005) and antimicrobial properties (Rojas et al., 2006) among others. These make annatto a very attractive and healthy pigment as a substitute for the many synthetic colorants.

The mechanism of bixin biosynthesis remains unresolved. However, it has been suggested that three genes govern bixin biosynthesis (lycopene cleavage dioxygenase, bixin aldehyde dehydrogenase, and norbixin carboxyl methyltransferase), which catalyze the sequential conversion of a $\mathrm{C}_{40}$ carotenoid, most probably lycopene, into bixin (Bouvier et al., 2003).

Annatto is currently cultivated in Africa, Asia, and Latin America (from Brazil to
Sample collection. Samples were collected between Oct. 2005 and Jan. 2006 from the locations of Cooperativa and Yaxcaba, Yucatan state, Mexico (Fig. 1). One to five panicles and five to 10 leaves from active shoots were collected from a total of 200 adult plants (100 in each Cooperativa and Yaxcaba). All plant materials were stored at $-80{ }^{\circ} \mathrm{C}$ until processing. In both locations, Cooperativa and Yaxcaba, the sample plants were 5- to 10-year-old seed-propagated plants. In Cooperativa, plants were grown in different fields, whereas in Yaxcaba, they were grown in the backyards of houses. The collected germplasm was chosen after review of available $B$. orellana materials in the herbarium at the Centro de Investigación Científica de Yucatán and based on the relationship between capsule morphology and bixin production (Rivera-Madrid et al., 2006). 


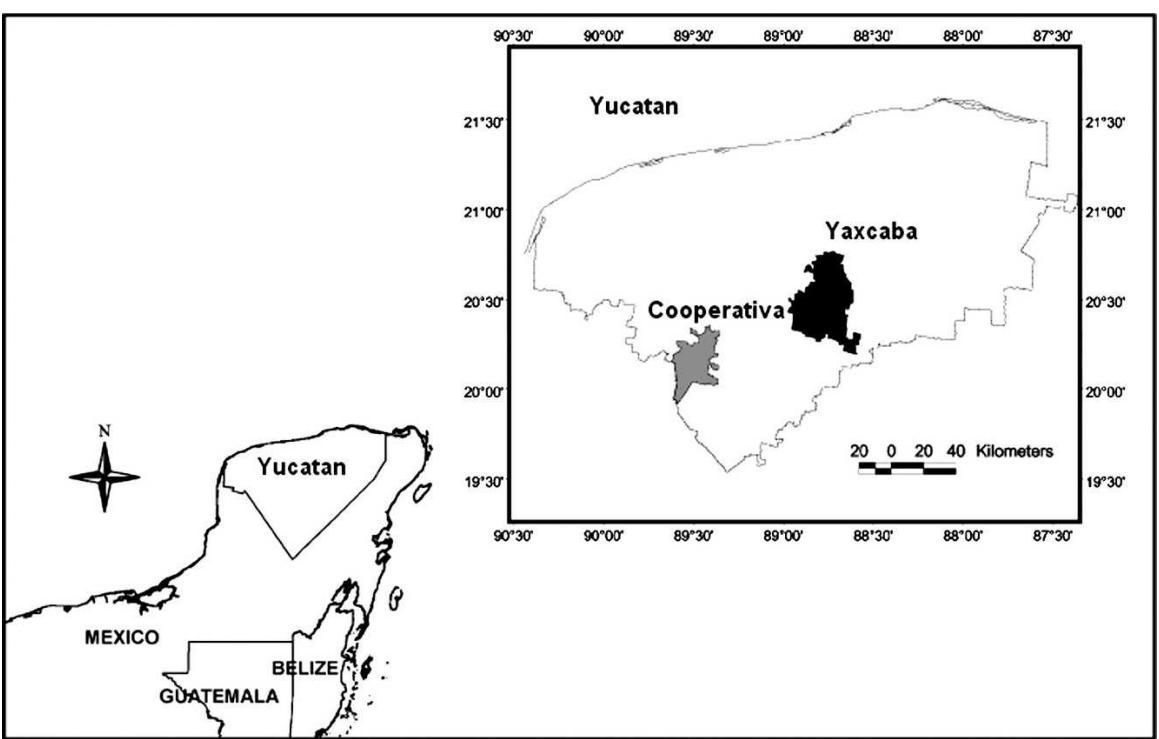

Fig. 1. Annatto accessions collection sites in Yucatan state, Mexico.

Morphological characterization. Thirteen quantitative and qualitative descriptors of the capsule, flower, and seed known to be important for annatto breeding were used in morphological characterization (Arce, 1999). The quantitative descriptors were: capsule length (cm; from tip to bottom); capsule width ( $\mathrm{cm}$; of widest part); seed number in five capsules; bixin content $\left(\mathrm{g} \cdot 100 \mathrm{~g}^{-1}\right)$; total carotene content $\left(\mathrm{g} \cdot 100 \mathrm{~g}^{-1}\right)$; capsule length/ width ratio; and bixin/carotene content ratio. The qualitative descriptors were: spine density $(0=$ no spines, $1=$ very low, $3=$ low, $5=$ medium, $7=$ high, $9=$ very high), spine length $(1=$ very short, $3=$ short, $5=$ medium, $7=$ long, $9=$ very long), apex shape $(1=$ rounded, 2 = acute, $3=$ acuminate, $4=$ chordate $)$, dehiscence $(1=$ dehiscent, $2=$ indehiscent $)$, flower color $(1=$ light pink, $2=$ pink, $3=$ purple, $4=$ fuchsia, $5=$ white $)$, and capsule color $(1=$ yellow, $2=$ green, 3 $=$ red, $4=$ dark red). All measurements were taken from mature materials, except for capsule color, which was recorded from immature materials. Measurements of 10 randomly chosen capsules were taken from each individual.

Seeds were placed in 2-mL plastic tubes, wrapped in aluminum foil to avoid contact with light and stored at $-20{ }^{\circ} \mathrm{C}$. Bixin content was determined in triplicate following Dendy's (1966) protocol, slightly modified for this study. A sample of $\approx 0.1 \mathrm{~g}$ was placed in a glass tube $(15 \mathrm{~mL})$ in an ice bath and $5 \mathrm{~mL}$ of chloroform was added and mixed for $1 \mathrm{~min}$ with an Ultra-turrax homogenizer (T25; Janke-Kunkel, Seelbach, Staufeni, Germany). The extract was filtered with a 25-mm Swinnex filter holder (Millipore, Billerica, MA; SX0002500, 0.45 mm; Millipore, Billerica, MA; HNWP02500), recovered in a plastic tube $(15 \mathrm{~mL})$, and the volume was adjusted to $5 \mathrm{~mL}$. A $10-\mu \mathrm{L}$ aliquot of bixin extract was diluted to $1 \mathrm{~mL}$ volume. Using a spectrophotometer (DU 600 Series; Beckman, Fullerton, CA), bixin was measured at a maximum absorbance of $500 \mathrm{~nm}$ and total $404 \mathrm{~nm}$. Absorbance records ranged from 0.5 to 1.0 to produce an accurate estimate of bixin content. Bixin and carotenoids contents were calculated using correction factors of 282.6 for bixin and 0.256 for total carotenoids.

Molecular characterization. A sample of $\approx 100 \mathrm{mg}$ of fresh leaf was ground in liquid nitrogen and total genomic DNA was extracted using a DNeasy Tissue Kit (Qiagen, Hilden, Germany; 69506). Following the method of Li and Quiros (2001), the SRAP protocol was implemented using 53 primer combinations to carotenoid content was measured at 500 and select for polymorphic bands. The selected primers consisted of $\approx 5$ polymorphic fragments from two individuals with contrasting traits, for example, large and dehiscent capsules with high bixin content or short and indehiscent capsules with low bixin content.

Twelve primer combinations were selected for SRAP analysis (Table 1). The forward primers were labeled with IRDye 800 or IRDye 700 . A standard polymerase chain reaction (PCR) cocktail mix was prepared according to Ruiz et al. (2005) and subjected to the following conditions: 1 cycle of $94{ }^{\circ} \mathrm{C}$ for $2 \mathrm{~min}, 5$ cycles of $94^{\circ} \mathrm{C}$ for $30 \mathrm{~s}$, $35^{\circ} \mathrm{C}$ for $30 \mathrm{~s}$, and $72{ }^{\circ} \mathrm{C}$ for $1 \mathrm{~min}$, and 35 cycles of $94{ }^{\circ} \mathrm{C}$ for $30 \mathrm{~s}, 50^{\circ} \mathrm{C}$ for $30 \mathrm{~s}, 72^{\circ} \mathrm{C}$ for $1 \mathrm{~min}$ followed by a final extension of $72{ }^{\circ} \mathrm{C}$ for $2 \mathrm{~min}$. Briefly, $3 \mu \mathrm{L}$ bidistilled water containing bromophenol blue $0.25 \%$ $(\mathrm{w} / \mathrm{v})$ and sucrose $40 \%(\mathrm{w} / \mathrm{v})$ was added to the PCR products before denaturing at $94{ }^{\circ} \mathrm{C}$ for $5 \mathrm{~min}$. After denaturing, $0.6 \mu \mathrm{L}$ of reaction mixture was loaded into $5.5 \%(\mathrm{w} /$ v) denatured acrylamide gels and continuous $1 \times$ TBE buffer. The samples were run in duplicate to ensure band pattern reproducibility. The PCR products were run in a sequencer (IR2 4200; LI-COR, Lincoln, NE).

Analysis of morphological data. A cluster analysis was performed to group the individuals from both studied sites according to similar morphological traits based on different quantitative and qualitative descriptors of the capsule, flower, and seed. To form groups and test for relationships between these groups and bixin content, a two-step cluster analysis algorithm was run using the SPSS 13.0 program (SPSS Inc., Chicago, IL). This is a relatively new cluster technique that can process both continuous and categorical variables with a log likelihood distance measure. It is therefore appropriate for forming groups according to the similarity of two variable types: quantitative variables (capsule length and width, length/width ratio, and number of seeds) and qualitative variables (spine density and length, capsule apex shape, flower and capsule color, and capsule dehiscence or indehiscence). The two-step cluster analysis consists of a preclustering step in which records are grouped into numerous small subclusters followed by a clustering step. The subclusters are grouped into clusters using an agglomerative hierarchical clustering approach. The method automatically

Table 1. Sequence-related amplified polymorphism primers used to develop the polymorphic markers used in the study.

\begin{tabular}{|c|c|c|c|}
\hline Name & Forward primers & Name & Reverse primers \\
\hline Odd-3 & $5^{\prime}$-CCAAAACCTAAAACCAGGA-3' & $\mathrm{Pm}-3$ & $5^{\prime}$-ACAAGATTTTGAGAATACTAC-3' \\
\hline Odd- 8 & 5'-CACAAGTCGCTGAGAAGG-3' & Odd- 15 & 5'-GCGAGGATGCTACTGGTT-3' \\
\hline Odd- 8 & 5'-CACAAGTCGCTGAGAAGG-3' & Odd- 21 & 5' -CAGAGTAATTGCCATGAAATG-3 \\
\hline Em-1 & 5'-GACTGCGTACGAATTCAA-3' & Odd- 45 & 5'-AAGTGAAGTGCCTACAATAC-3' \\
\hline Em-1 & 5'-GACTGCGTACGAATTCAA-3' & Odd- 21 & 5' -CAGAGTAATTGCCATGAAATG-3 \\
\hline Em-2 & 5'-GACTGCGTACGAATTCTG-3' & Me-2 & 5'-TGAGTCCAAACCGGAGC-3' \\
\hline $\mathrm{Me}-2$ & 5'-TGAGTCCAAACCGGAGC-3' & Odd-2 & 5'-TTAGTCTTCACTTCCAAACC-3' \\
\hline $\mathrm{Me}-2$ & 5'-TGAGTCCAAACCGGAGC-3' & Sa-9 & $5^{\prime}$-GTTGAGAGTGTTGATTGG T-3' \\
\hline
\end{tabular}


chooses the optimum number of clusters by examining the Akaike information criterion values (Chiu et al., 2001).

Significant differences between the groups produced in the two-step cluster analysis were evaluated with an analysis of variance (ANOVA) and Tukey's honestly significant difference (HSD) test for numerical variables and Pearson's $\chi^{2}$ and Fisher's exact test for categorical variables. Another ANOVA was run to analyze differences in bixin content among collection sites in each group as well as between groups.

Analysis of genetic data. Each SRAP fragment was coded " 1 " for the presence of a specific allele or " 0 " in its absence. Similarity matrices based on Nei and Li's similarity coefficient was used to construct an unweighted pair group method with arithmetic average dendrogram using NTSYS-PC version 2.02 (ExeterSoftware, Setauket, NY). Statistical stability of the branches in the dendrogram was tested by bootstrap analysis with 1000 replicates using the Free Tree 0.9.1.50 software program (Pavlicek et al., 1999). A specific B. orellana variant (pink flower, high bixin content, red and dehiscent capsule) was used as a positive control to confirm any polymorphic bands detected in the $B$. orellana samples.

An analysis of molecular variance (AMOVA) (Excoffier et al., 1992) was used to determine molecular variance between and within collection sites using the Arlequin 3.1 software (Excoffier et al., 2005). The significance of variance component $\Phi$ statistics was tested using 1000 permutations of a nonparametric permutation approach described in Excoffier et al. (1992). The level of genetic differentiation between samples was analyzed by calculating pairwise $\Phi_{\mathrm{ST}}$ estimations.

\section{Results}

Morphological analysis. These analyses were performed on samples from 87 individuals of $B$. orellana plants, 42 from Cooperativa and 45 from Yaxcaba. Three groups with similar morphological characteristics were recognized from 87 individuals of $B$. orellana based on the two-step cluster analysis of quantitative and qualitative descriptors. Group 1 individuals (seven from Cooperativa and 11 from Yaxcaba) had larger and narrower capsules, i.e., a larger capsule and shorter length/width ratio than individuals in Group 2 (21 from Cooperativa and 28 from Yaxcaba) and Group 3 (14 from Cooperativa and six from Yaxcaba). The only difference between individuals in Groups 2 and 3 was capsule width with Group 2 having wider capsules than Group 3 (Fig. 2). A large proportion of Group 1 individuals had capsules with short to medium spines, a rounded and acuminate capsule apex, purple flowers, and red and dark red capsules that were dehiscent. In contrast, most individuals in Groups 2 and 3 had capsules with large to very large spines, an agreed capsule apex, white flowers, and yellow and green capsules that were indehiscent (Fig. 3).

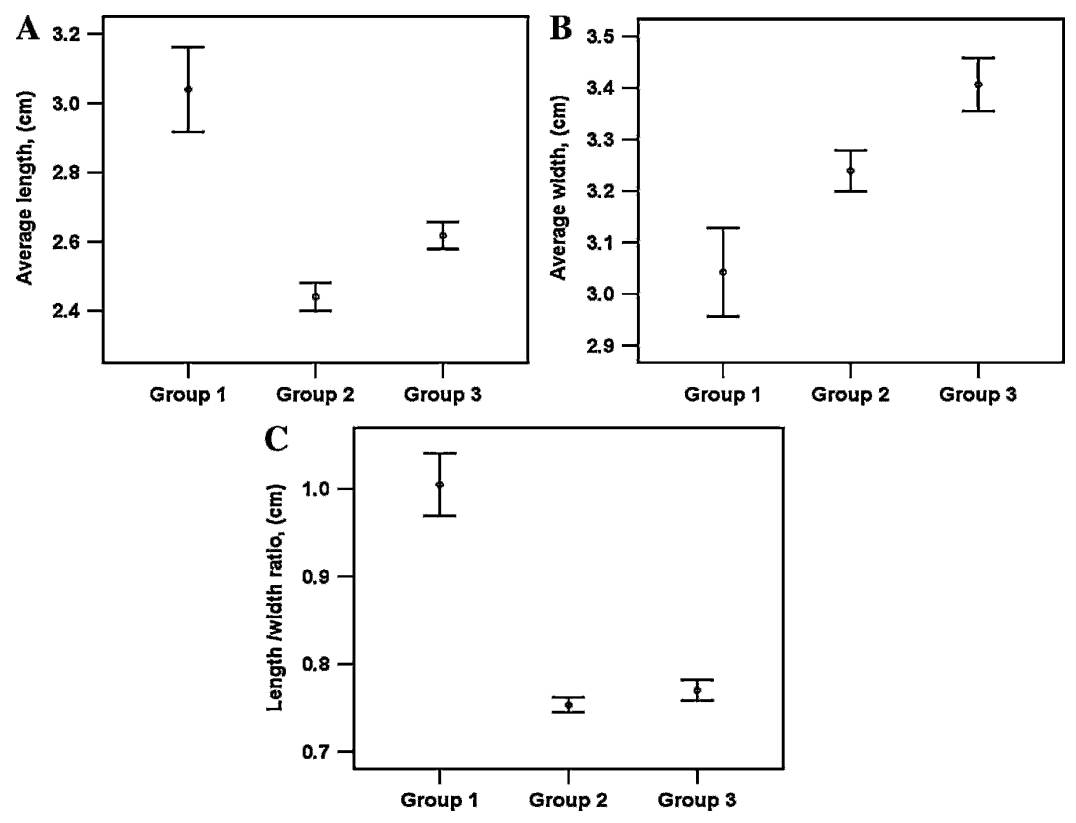

Fig. 2. Differences in capsule size between clustered groups: $(\mathbf{A})$ average length $(\mathrm{cm})\left(\mathrm{F}_{[2,86]}=21.85, P<\right.$ 0.000 ; Tukey honestly significant difference (HSD) at $5 \%)$; (B) average width $(\mathrm{cm})\left(\mathrm{F}_{[2,86]}=7.56, P<0\right.$. 001; Tukey HSD at $5 \%)$; and $(\mathbf{C})$ length/width ratio $(\mathrm{cm})\left(\mathrm{F}_{[2,86]}=59.39, P<0.000\right.$, Tukey HSD at $\left.5 \%\right)$.
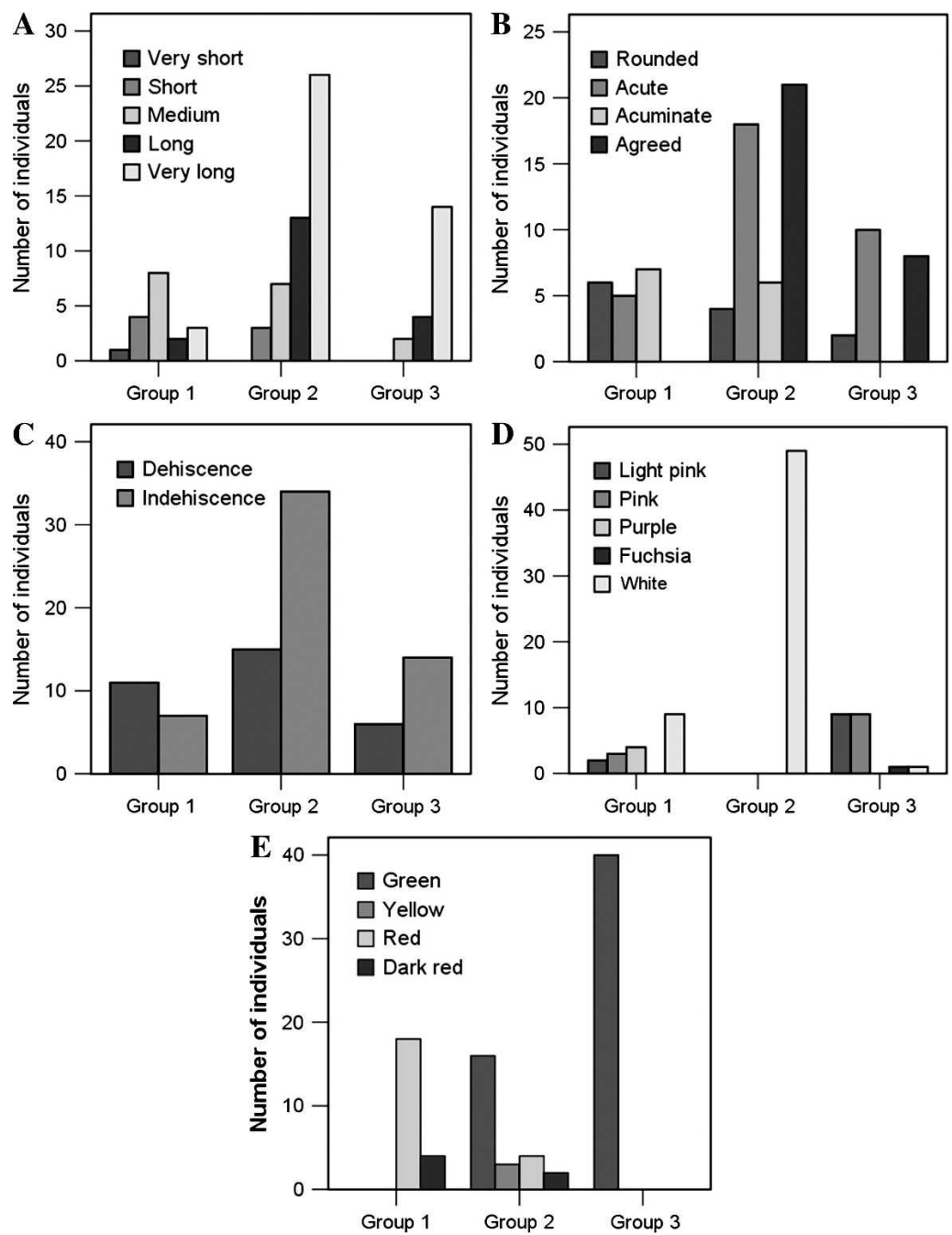

Fig. 3. Differences in qualitative morphological features between clustered groups: (A) capsule spine length (Fisher's exact test $=21.63, P<0.001) ;($ B $)$ capsule apex shape (Fisher's exact test $=25.66, P<$ 0.000 ); (C) capsule dehiscence (Pearson's $\chi^{2}=5.78, P<0.058$ ); (D) flower color (Fisher's exact test $=$ 77.66, $P<0.000$ ); and $(\mathbf{E})$ capsule color (Fisher's exact test $=80.57, P<0.000$ ). 
Average bixin content was highest for Group 1 individuals from Cooperativa (Table $2, \mathrm{~F}_{[2,41]}=10.59, P<0.001$; Tukey HSD, $P<$ $0.001)$. Average bixin content of individuals from Yaxcaba did not differ between groups (Table 2, $\mathrm{F}_{[2,44]}=0.795 ; P<0.458$ ).

Genetic analysis. Using the same 87 individuals as in the morphologic analysis, and 12 polymorphic primer combinations, a total of 58 reproducible bands were detected with an average of six bands and a range of one to 11 bands per primer set.

Two large clusters were defined in the dendrogram (Fig. 4): Cluster I grouped all individuals from Cooperativa and Cluster II grouped all those from Yaxcaba. According to similarity distances, both clusters exhibited high genetic variation: Cluster I exhibited the highest genetic variation (0.14815 to 0.8679 ) compared with Cluster II (0.42553 to 0.97143$)$. No duplicates were detected among the analyzed individuals. An AMOVA also showed genetic variation to be attributed to differences between $(61.55 \%)$ and within sampling sites $(38.45 \%)$. A significant $\Phi$ value $\left(\Phi_{\mathrm{ST}}=0.61, P<0.00\right)$

Table 2. Mean bixin content $( \pm \mathrm{SE})$ in morphological groups produced in the cluster analysis by site.

\begin{tabular}{llc}
\hline Morphological & \multicolumn{2}{c}{ Collection sites } \\
\cline { 2 - 3 } groups & Cooperativa & Yaxcaba \\
\hline Group 1 & $2.65 \pm 0.24^{\mathrm{z}, \mathrm{y}}$ & $1.83 \pm 0.14^{\mathrm{z}}$ \\
Group 2 & $1.84 \pm 0.08^{\mathrm{y}}$ & $1.72 \pm 0.07$ \\
Group 3 & $1.66 \pm 0.15^{\mathrm{y}}$ & $1.92 \pm 0.08$ \\
\hline
\end{tabular}

zSignificant differences were found between sites in these groups $(P<0.001)$.

${ }^{y}$ Significant differences were found between groups in these collection sites $(P<0.001)$. further confirmed the differentiation between Cooperativa and Yaxcaba.

\section{Discussion}

General. The morphological analysis clustered individuals with desirable characteristics for genetic improvement of $B$. orellana in Group 1. Capsule size was not found to affect seed number, although previous reports have suggested that large, wide capsules are positively correlated with seed size (Medina et al., 2001b). Group 1 also had capsules with short to intermediate length spines, which is a desirable trait because it facilitates capsule harvest and seed recovery (Medina et al., 2001b) (Fig. 3A). The pink or purple flower color and red capsule color of this group (Fig. 3D-E) are associated with higher bixin content (Table 2). Groups 2 and 3 had white flowers and yellow and green capsules (Fig. 3D-E) as well as lower bixin content (Table 2). This coincides with RiveraMadrid et al. (2006), who reported that individuals with purple flowers and red capsules had higher bixin content than those with white flowers and green capsules. Another advantage of individuals with purple or pink flowers is that outcrossed hybrids exhibit higher fruit set than those of white flower individuals (Rivera-Madrid et al., 2006). Parents with purple or pink flowers and red capsules are therefore potential sources for genetic improvement of annatto with the goal of increasing bixin content.

The difference in bixin content between Group 1-Cooperativa and Group 1-Yaxcaba individuals and Group 2 and 3 individuals is probably the result of the different uses of annatto seeds at each site. In Cooperativa,

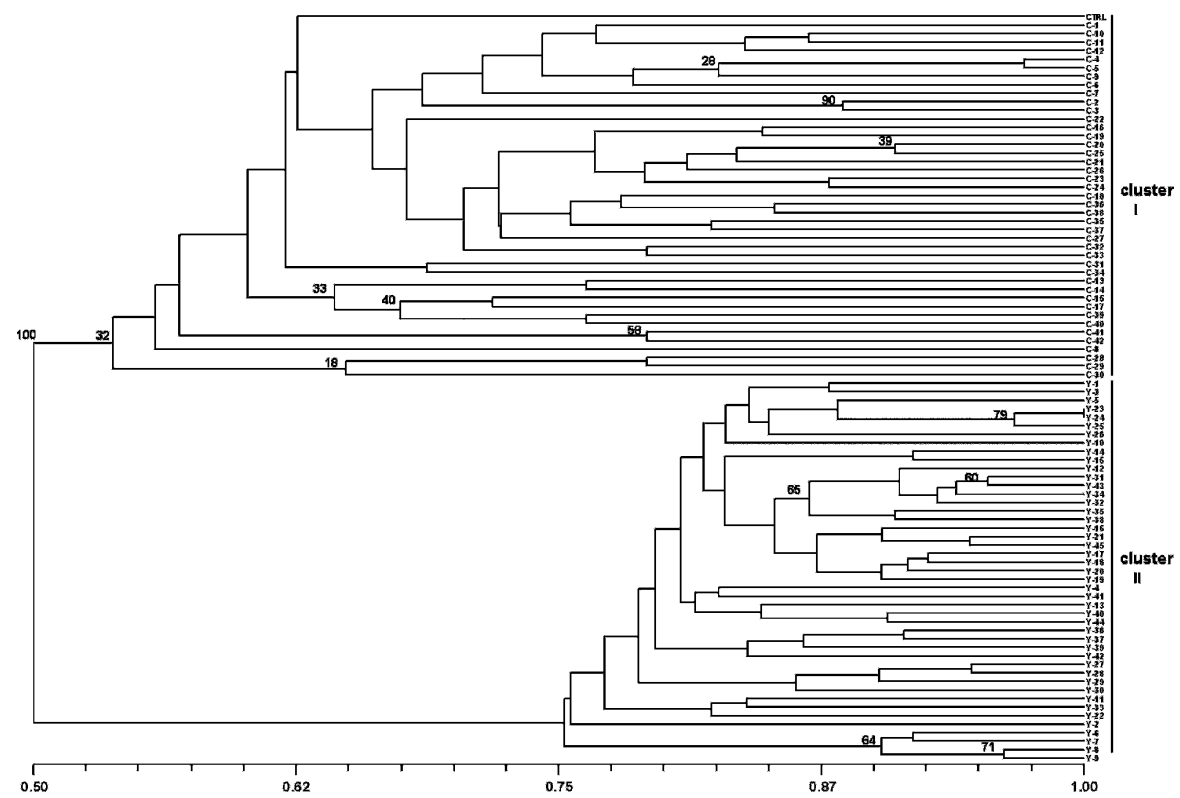

Fig. 4. Dendrogram of annatto collection sites based on unweighted pair group method with arithmetic average cluster analysis of 58 sequence-related amplified polymorphism (SRAP) molecular markers in the study. The resulting clusters are labeled Clusters I and II. The letter " $\mathrm{C}$ " indicates an individual collected at Cooperativa and "Y" one collected at Yaxcaba. CTRL indicates the positive control (pink flower, high bixin content, red and dehiscent capsule). The numbers at the nodes indicate the percentage bootstrap values.

seeds are probably selected for commercial use in bixin extraction, whereas in Yaxcaba, the seeds are for household use and may not be subject to heavy selection pressure.

Individuals in Groups 2 and 3 had indehiscent capsules (Fig. 3C). This is an important breeding target, because indehiscence may enhance crop yields (Roberts et al., 2002), and dehiscent capsules expose seeds to sunlight, leading to bixin photo-oxidation and decreased bixin content (Rivera-Madrid et al., 2006). The present results coincide with Mazzani et al. (2000) (Fig. 3B-C). The genetic basis for dehiscence and indehiscence capsule in $B$. orellana is not known. In Brassica and Arabidopsis thaliana, dehiscence is controlled by the $M A D S$ gene family (Liljegren et al., 2000).

Genetic improvement of annatto is currently incipient and largely empirical. Some effort has been made to select for high bixin content and traits that favor bixin yield such as pod indehiscence, flower color, and so on. Molecular markers have not been used to investigate the genetic variability of Bixa orellana instead the SRAP molecular markers were used to confirm the high observed morphological variation shown here, because this method is powerful enough to detect sequences codifying for morphological characters (Espósito et al., 2007; Ruiz et al., 2005). Based on the similarity genetic diversity index, all individuals have sufficient variation to permit genetic improvement by interindividuals.

The dendrogram built from the genetic analysis showed that the individuals studied in this research were clustered by collection site rather than morphological characters (Fig. 4). The AMOVA supported the dendrogram results by indicating there to be high genetic differentiation between and within collection sites. This was confirmed by the genetic distances among Cooperativa individuals and among Yaxcaba individuals as well as between the sites. This differentiation is probably the result of Cooperativa farmers introducing seed from stock with commercially desirable traits and from variable sources, whereas in Yaxcaba, seeds were probably introduced independently by different households at different times.

In summary, genetic analysis indicated that both sampled sites contain different annatto accessions, implying that they probably originated from independent introductions from different locations. This high genetic variability in all individuals expands the gene pool and increases the possibility of selecting elite parents for genetic improvement. In particular, Group 1 exhibited desirable morphological traits that make it a promising source for selection of individuals with traits linked to high pigment yield. These could then be included in a breeding and clonal propagation program focused on establishment of new plantations. The undesirable trait of capsule dehiscence could be mitigated by crossing blocks with Groups 2 and 3 individuals with indehiscent capsules. As reported by Medina et al. (2001b), large and 
indehiscent capsules without spines are desirable because they facilitate harvest and produce higher bixin content. Genetically diverse parents may also manifest a broad range of phenotypic variation potentially useful for further trait selection (McCouch, 2004). Future research can make genetic improvement of annatto more efficient by focusing on segregation of contrasting traits from crossing individuals to map genes of interest for marker-assisted selection of useful traits. Genetic analysis can also be used to identify DNA markers linked to quantitative trait loci affecting important traits in the $B$. orellana genome.

\section{Literature Cited}

Arce, J. 1999. El achiote Bixa orellana L. cultivo promisorio para el trópico. EARTH Press, Costa Rica.

Bouvier, F., O. Dogbo, and B. Camara. 2003. Biosynthesis of the food and cosmetic plant pigment bixin (annatto). Science 300:2089-2091.

Chiu, T., D. Fang, J. Chen, Y. Wang, and C. Jeris. 2001. A robust and scalable clustering algorithm for mixed type attributes in large database environment. Proc. of the Seventh ACM SIGKDD international conference on knowledge discovery and data mining, San Francisco, CA.

Dendy, D.A.V. 1966. The assay of annatto preparations by thin-layer chromatography. J. Sci. Food Agr. 17:216-218.

Espósito, M.A., E.A. Martin, V.P. Cravero, and E. Cointry. 2007. Characterization of pea accessions by SRAP's markers. Sci. Hort. 113:329335.

Excoffier, L., G. Laval, and S. Schneider. 2005. Arlequin ver. 3.0: An integrated software package for population genetics data analysis. Evolutionary Bioinformatics 1:47-50.

Excoffier, L., P.E. Smouse, and J.M. Quattro. 1992. Analysis of molecular variance inferred from metric distances among DNA haplotypes: Application to human mitochondrial DNA restriction data. Genetics 131:479-491.

Giuliano, G., C. Rosati, and P. Bramley. 2003. To dye or not to dye: Biochemistry of annatto unveiled. Trends Biotechnol. 21:513-515.

Júnior, A.C.T.S., L.M.B.O. Asad, E.B. De-Oliveira, K. Kovary, N.R. Asad, and I. Felzenszwalb. 2005. Antigenotoxic and antimutagenic potential of an annatto pigment (norbixin) against oxidative stress. Genet. Mol. Res. 4:94-99.

Li, G. and C.F. Quiros. 2001. Sequence-related amplified polymorphism (SRAP), a new marker system based on a simple PCR reaction: Its application to mapping and gene tagging in Brassica. Theor. Appl. Genet. 103:455-461.

Liljegren, S.J., G.S. Ditta, Y. Eshed, B. Savidge, J.L. Bowman, and M.F. Yanofsky. 2000 SHATTERPROOFMADS-box genes control seed dispersal in Arabidopsis. Nature 404: 766-770.

Mazzani, E., R.C. Marín, and V. Segovia. 2000. Estudio de la variabilidad existente en la colección de onoto (Bixa orellana L.) del CENIAP, FONAIAP. Venezuela. Rev. Fac. Agron. 17:492-504. (LUZ).

McCouch, S. 2004. Diversifying selection in plant breeding. PLoS Biol. 2:1507-1512.

McGonigle, K.E.M. and K.J. McCracken. 2002. Study on the effects of annatto (Bixa orellana) and Yucca Schidegera on cholesterol content and yolk colour. Br. Poult. Sci. 43:S41-S42.

Medina, A.M., C. Michelangeli, C. Ramis, and A. Díaz. 2001a. Identificación y variabilidad genética de genotipos de onoto (Bixa orellana L.) mediante el uso de proteínas hidrosolubles e isoenzimas. ACV 52:24-33.

Medina, A.M., C. Michelangeli, C. Ramis, and A. Díaz. 2001b. Caracterización morfológica de frutos de onoto (Bixa orellana L.) y su correspondencia con patrones de proteínas e isoenzimas. ACV 52:14-23.

Pavlicek, A., S. Hrda, and J. Flegr. 1999. FreeTree: Freeware program for construction of phyloge- netic trees on the basis of distance data and bootstrap/jackknife analysis of the tree robustness. Application of the RAPD analysis of genus Frenkelia. Folia Biol. (Praha) 45:97-99.

Poppendieck, H.-H. 2001. Bixaceae. In: Stevens, W.D., U.C. Ulloa, A. Pool, and O.M. Montiel (eds.). Flora de Nicaragua. Introducción gimnospermas y angiospermas (AcanthaceaeEuphorbiaceae). Missouri Botanical Garden Press, St. Louis, MO

Portela de Carvalho, J.F.R., I.P. Robinson, and A.C. Alfenas. 2005. Isozymic variability in a Brazilian collection of annatto (Bixa orellana L.). Pesqui. Agropecu. Bras. (Brasília) 40:653660.

Rivera-Madrid, R., R.M. Escobedo-GM, E. BalamGalera, M. Vera-Ku, and H. Harries. 2006. Preliminary studies toward genetic improvement of annatto (Bixa orellana L.). Sci. Hort. 109:165-172.

Roberts, J.A., K.A. Elliott, and Z.H. GonzalezCarranza. 2002. Abscission, dehiscence, and other cell separation processes. Annu. Rev. Plant Biol. 53:131-158.

Rojas, J.J., V.J. Ochoa, S.A. Ocampo, and J.F. Muñoz. 2006. Screening for antimicrobial activity of ten medicinal plants used in Colombian folkloric medicine: A possible alternative in the treatment of non-nosocomial infections. BMC Complement. Altern. Med. 6:1-6.

Ruiz, J.J., S. García-Martínez, B. Picó, M. Gao, and C.F. Quiros. 2005. Genetic variability and relationship of closely related spanish traditional cultivars of tomato as detected by SRAP and SSR markers. J. Amer. Soc. Hort. Sci. 130:88-94.

Russell, K.R.M., E.Y.S.A. Morrison, and D. Ragoobirsingh. 2005. The effect of annatto on insulin binding properties in the dog. Phytother. Res. 19:433-436.

Sandy-Cuen, P.M. and R. Becerra. 2003. Manejo campesino de recursos naturales. El achiote. BioDiversitas 7:7-11. 